\title{
Numerical Visualization of Fluid Flow and Filtration Efficiency in Centrifugal Oil Purifier
}

\author{
Ho-Yun Jung ${ }^{1} \cdot$ Yoon-Hwan Choi $^{2} \cdot$ Yeon-Won Lee ${ }^{\dagger} \cdot$ Deog-Hee Doh $^{3}$ \\ (Received January 8, 2010; Revised January 25, 2010; Accepted 26, 2010)
}

\begin{abstract}
The centrifugal oil purifier is used in ships for purifying the engine lubrication oil. The momentum needed for the rotation of the cylindrical chamber is obtained by jet injections. The dust particles in the oil are separated by the centrifugal forces moving to the inner wall of the rotating cylindrical chamber body. The dust particles are eliminated when the particles are adsorbed onto the surface of the inner wall of the chamber body. The flow characteristics and the physical behaviours of particles in this centrifugal oil purifier have been investigated numerically and the filtration efficiencies have been evaluated. For the calculations, a commercial code has been used and the SST k- $\omega$ turbulence model has been adopted. The MRF (Multiple Reference Frame) method has been introduced to consider the rotating effect of the flows. Under various variables, such as particle size, particle density and rotating speed, the filtration efficiencies have been evaluated. It has been verified that the filtration efficiency is increased with the increments of the particle size, the particle density and the rotating speed of the cylindrical chamber.
\end{abstract}

Key words : Centrifugal Oil Purifier, Filtration Efficiency, CFD(Computational Fluid Dynamics), MRF (Multiple Reference Frame)

\section{Nomenclature}

$F_{B}$ : Buoyancy force due to gravity [N]

$F_{B A}$ : Basset force or history term [N]

$F_{D}$ : Drag force acting on the particle [N]

$F_{P}$ : Pressure gradient force [N]

$F_{R}$ : Forces due to domain rotation [N]

$F_{V M}:$ Added mass force $\left.\llbracket \mathrm{N}\right\rfloor$

\section{Greek Symbols}

$\rho$ : Density of particle $\left[\mathrm{kg} / \mathrm{m}^{3}\right]$

$\eta$ : Filtration efficiency [\%]

$\mu$ : Viscosity of working oil $[\mathrm{kg} / \mathrm{s} \cdot \mathrm{m}]$

\section{Introduction}

An oil purifier is one of the key components in marine diesel engines. Generally, FO(Fuel Oil) purifier, LO (Lubrication Oil) purifier and system oil filter have been widely used for maintaining clean condition in marine engines. The extension of life expectancy of oil is strongly required due to soaring oil price. As a result, the importance of the purifier is rising.

Figure 1 shows a schematic diagram of circulation system of lubrication oil. Most

\footnotetext{
† Corresponding Author(School of Mechanical Engineering, Pukyong National University, E-mail:ywlee@pknu.ac.kr, Tel:051-629-6162)

1 School of Mechanical Engineering, Graduated School, Pukyong National University

2 BK21, School of Mechanical Engineering, Pukyong National University

3 Division of Mechanical and Information Engineering, Korea Maritime University
} 
of the purifiers used for the full flow filter.

This filter belongs to element types, and this kind of filters can't purify the sub-micron sized matters in the oil. In order to purify such matters the pore size should be reduced in the conventional filters. However, this produces large pressure drops, which eventually produces the oil circulations worse in the main lubricating lines. To solve this problem, a centrifugal oil purifier which takes about $10 \%$ of the total circulating oil from the main oil circulation system for purification has been used[1].

There have been several studies on the centrifugal type filters. Jeong[2] carried out a study on the standard criteria of solid particle separation test for marine centrifugal purifier. Lee and $\operatorname{Kim}[3]$ carried out a basic study on the integrated lubrication system for large scale marine diesel engine in terms of the lubrication efficiencies. Kim et al. [4] studied on the enhancements of the filtration efficiencies of the centrifugal purifiers in case of gas-liquid two phase flows. Bang et al.[5] evaluated the flow characteristics and the filtration efficiencies of a cylindrical centrifugal purifier for the case with simple inlet and outlet conditions. Smiles[6] investigated the centrifugal filtration of particulate systems by numerical analysis. Most of the researches have been focused on the cases having simple inlet and outlet conditions not having actual using conditions.

The purpose of this study is to investigate the flow characteristics inside of the centrifugal oil purifier and the movements of the impurity matters for the case of actual using conditions. Further, the effects of number of blades, particle density, particle size and rotating speed of the filter have been also investigated in terms of filtration efficiency.

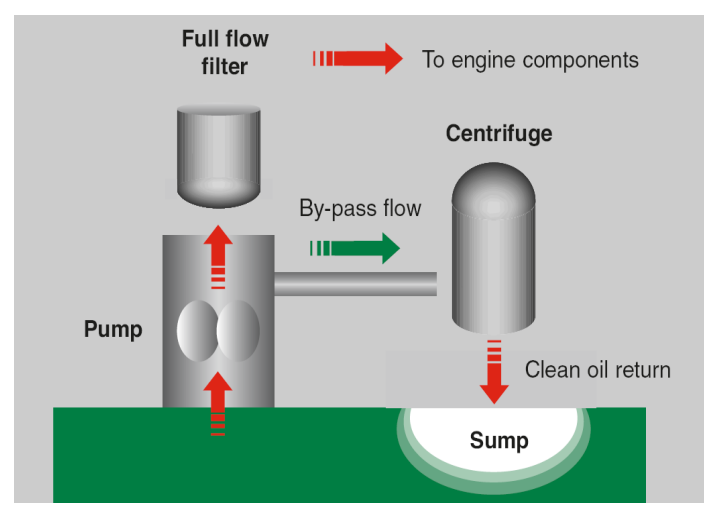

Figure 1: Schematic diagram of circulation system of lubrication oil (cited from MANN+HUMMEL corporation)

\section{Numerical Analysis}

Figure 2 shows the cross sectional view of the centrifugal oil purifier. The oil comes in from the inlet at the upper part of the rotating shaft and it goes out via the outlet at the lower part. The oil inside of the purifier is accelerated with the centrifugal force and the impurity matters are separated from the oil due to the inertia of their mass and move toward the inner surface of the rotating cylinder, and are lastly accumulated at the wall of the purifier where a sheet of paper filter is installed. In a mean while, the centrifugal force is produced by the rotational moments which are generated by injecting oil via two nozzles installed at the lower part of the purifier. 


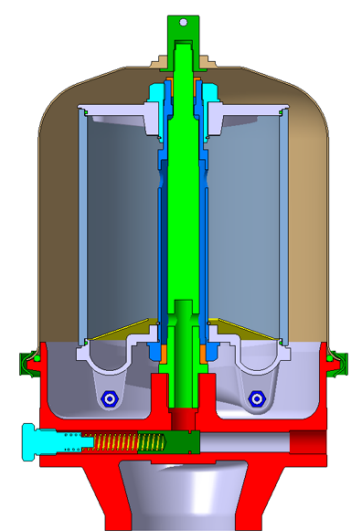

Figure 2: Schematic diagram of centrifugal oil purifier

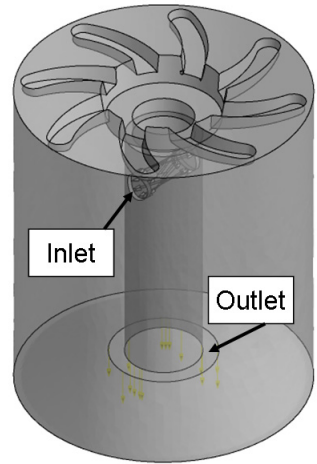

Figure 3: Analysis domain for simulation (8 blade model)

Figure 3 shows the calculation domain for the analyses. The grid system, the tetrahedral mesh, has been used and the prism layer has been constructed for the wall side. The number of the grids is 700,000 cells. In this study, three types of blade models, 4-blade, 8-blade, and 12-blade models, have been tested. Fig. 4 shows the shapes of 4-blade and 12-blade models.

The physical properties of the operating oil tested (SAE 30) are the temperature of $90^{\circ} \mathrm{C}$, the density $\rho=853.9 \mathrm{~kg} / \mathrm{s}$, and the viscosity $\mu=0.00356 \mathrm{~kg} / \mathrm{s} \cdot \mathrm{m}$.

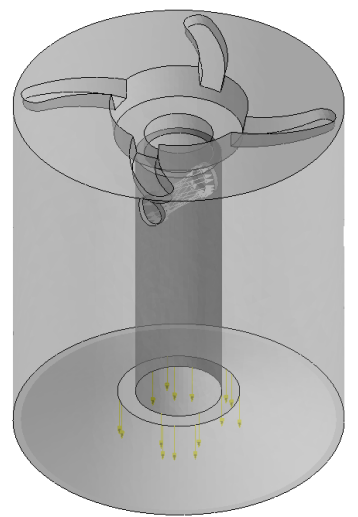

(a) 4 blades

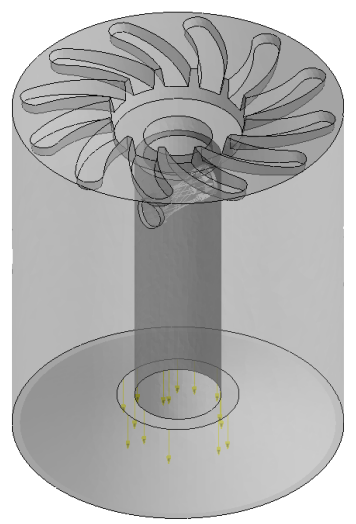

(c) 12 blades

Figure 4: Tested models

The SST (Shear Stress Transport) turbulence model was used, because that it was known to superior to standard $k-\epsilon$ and $k-\omega$ models for the flows having higher shear stresses. For analyzing the rotational effect of the flow fields, the function of MRF (Multiple Reference Frame) has been applied and the equilibrium equation for the force acting on the particles has been presented by Lagrangian coordinate system depicted as the following equation (1). This equation had been derived by Basset, Boussinesq and Oseen for a rotating reference frame[7]. 


$$
m_{P} \frac{d U_{P}}{d t}=F_{D}+F_{B}+F_{R}+F_{V M}+F_{P}+F_{B A}
$$

For calculating the movements of particles, flow field analyses has been performed first, and from the results the motions of the discrete particles have been predicted based on the phase model in which the behavior of particles are calculated. In this study, the interactions between the dust particles and the working fluids have been neglected because the particle sizes are small enough not influencing the working fluids. The forces acting on the particles have been calculated from the pressure gradients. The number of used grid for calculation has been decided at the condition that there is no grid dependency. The validation for the numerical simulation is now being tested experimentally for the next research reports.

\section{Results and Discussion}

The filtration efficiency has been defined by the ratio of the particle numbers filtrated over the total particle numbers inserted into the purifier. Accordingly, the filtration efficiency can be described as the following Eq. (2).

$$
\begin{aligned}
& \text { Filtration Efficiency }(\eta)= \\
& \frac{\text { Particle umbers filtrated }}{\text { Total particle } \nu \text { mbers inserted }} \times 100(\%)
\end{aligned}
$$

Figure 5 shows streamlines of the flow fields. It shows a little complex flow pattern caused by the effects of the blades. The fluid injected from the inlet moves along the streamline and the rising flow is created owing to the blade effects. The influences of particle density, particle size and rotating speed of each model (4,
8, 12 blades) have been explained in Fig.

6, Fig. 7 and Fig. 8.

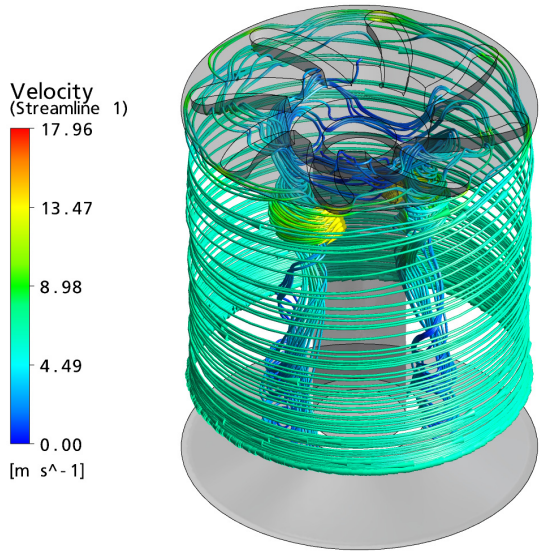

Figure 5: Streamlines of flow field (at $3500 \mathrm{rpm}, 8$ blades model)

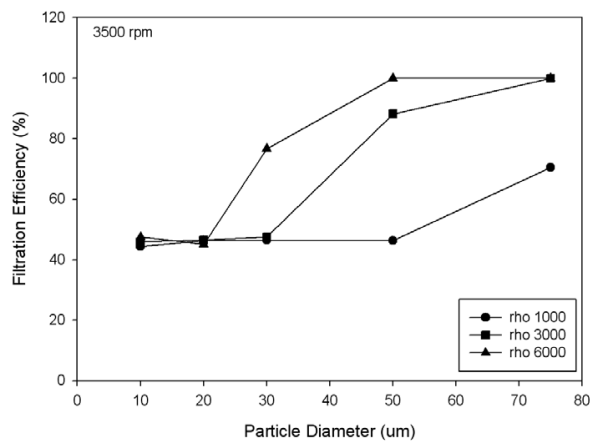

(a) effects of particle size and density

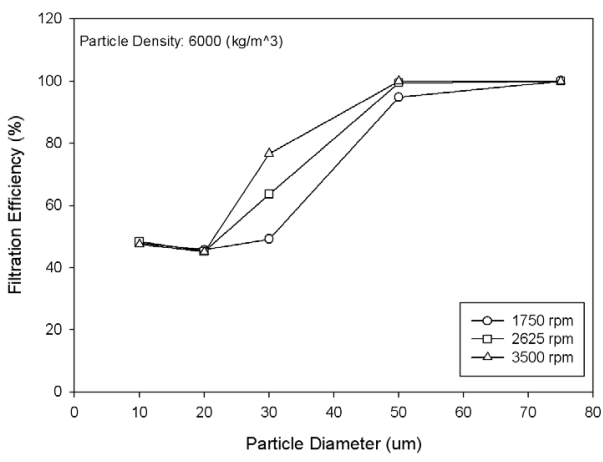

(b) effects of particle size and rpm

Figure 6: Filtration efficiency for the various conditions at 8 blade case 
Figure 6(a) shows the filtration efficiency according to the density and the size of the particle. Under high density of particle, the filtration efficiency is higher, and its tendency becomes larger at smaller particle sizes. This can be inferred from Eq.(1) that the radial force due to the buoyance force $\left(F_{B}\right)$ becomes larger than the tangential force due to the drag force $\left(F_{D}\right)$.

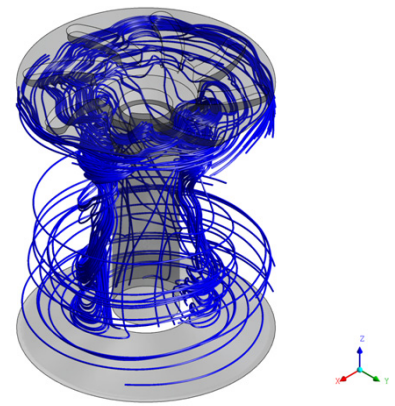

(a) $1750 \mathrm{rpm}$

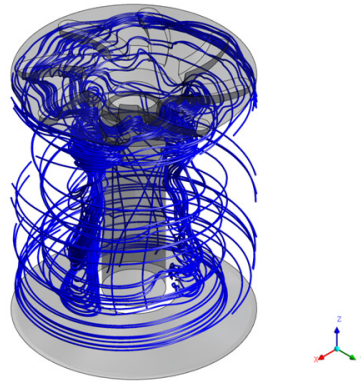

(b) $2625 \mathrm{rpm}$

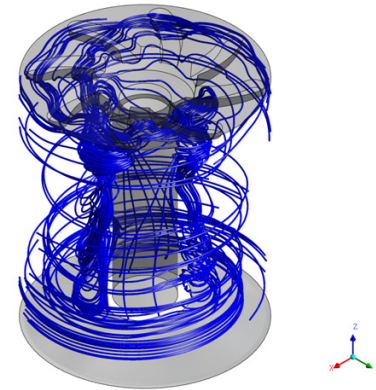

(c) $3500 \mathrm{rpm}$

Figure 7: Physical behavior of particles along the rotating speed of 8 blade model (particle $\rho=6000$ $\mathrm{kg} / \mathrm{m} 3$, size $=30 \mu \mathrm{m})$

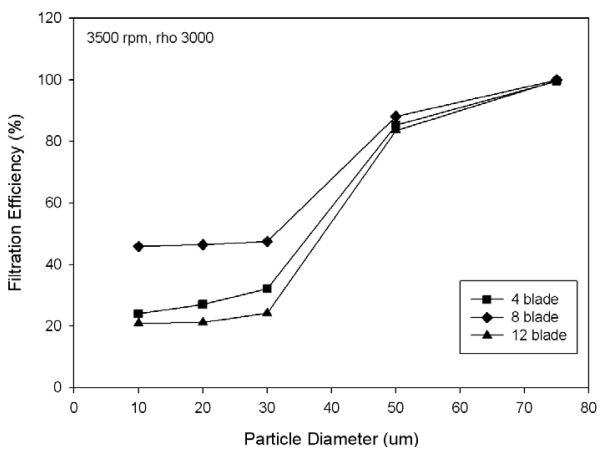

Figure 8: Filtration efficiency for the number of blades

In case of the particle density $\rho=6000$ $\mathrm{kg} / \mathrm{m} 3$, the efficiency begins increasing at the size of $30 \mu \mathrm{m}$. In case of the particle density $\rho=1000 \mathrm{~kg} / \mathrm{m} 3$, the efficiency begins increasing at the size of $75 \mu \mathrm{m}$. Figure 6(b) shows the filtration efficiency according to the rotation speed[rpm] and the size of the particle. The efficiency increased with increments of the rotating speed. At the particle's size $30 \mu \mathrm{m}$, the efficiency showed largely differences according to the rotating speed. When the particle's size is bigger than $50 \mu \mathrm{m}$ and is smaller than $20 \mu \mathrm{m}$, the efficiencies do not show any dependency on the rotating speed. This implies that the blades installed near the upper cover induce the flow to upper radial direction, and the particles in the flow travel along the outer wall of the rotating chamber, and they are lastly eliminated by being attached upon the filter paper.

Figure 7 shows the effect of rotating speed to the behaviors of particles in case of 8 blades with $\rho=6000 \mathrm{~kg} / \mathrm{m} 3$ and $\mathrm{d}=30 \mu$ $\mathrm{m}$. When the rotating speed is high(at $3500 \mathrm{rpm})$, it can be seen that the ending points of the particles' trajectories become 
disappeared, which implies that many particles are eliminated by the paper filter installed at the outer wall of the rotating chamber.

Figure 8 shows the effect of the number of blades to the filtration efficiency under the condition of $3500 \mathrm{rpm}$ and $\rho=3000$ $\mathrm{kg} / \mathrm{m} 3$ of particle density. In case of the 8-blade, the highest efficiency is seen among the three cases for all particle sizes. However, the differences of the efficiencies become small over the size $50 \mu$ $m$, which implies that the effects of the blade number to the efficiency is not seen any more over a certain number of blades. This sort of result can be found in the studies on the centrifugal pumps. According to the studies done by Oztekin et al.[8] and Stepanoff et al.[9], the optimal number of blades is 5 to 9 for the
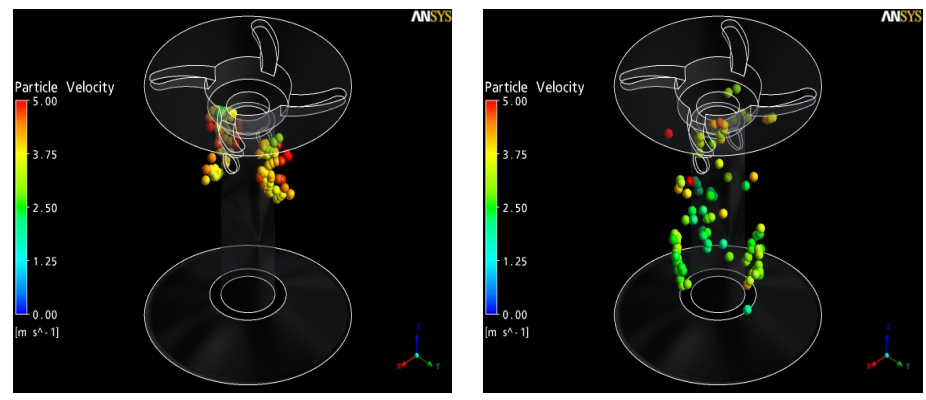

(a) 4 blade
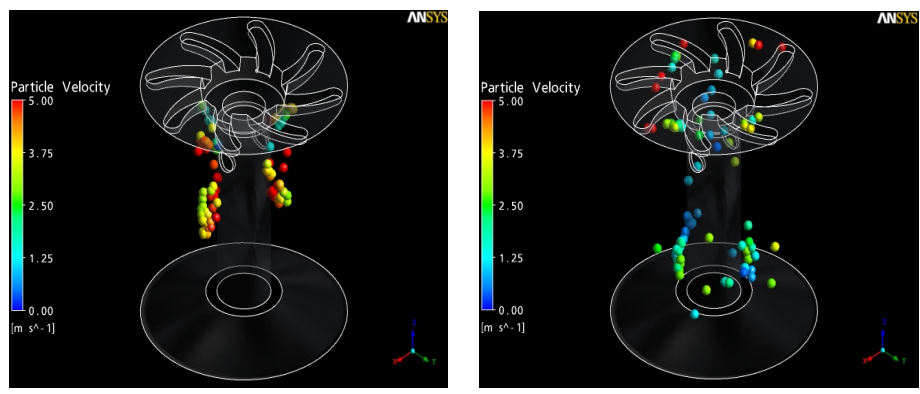

(b) 8 blade
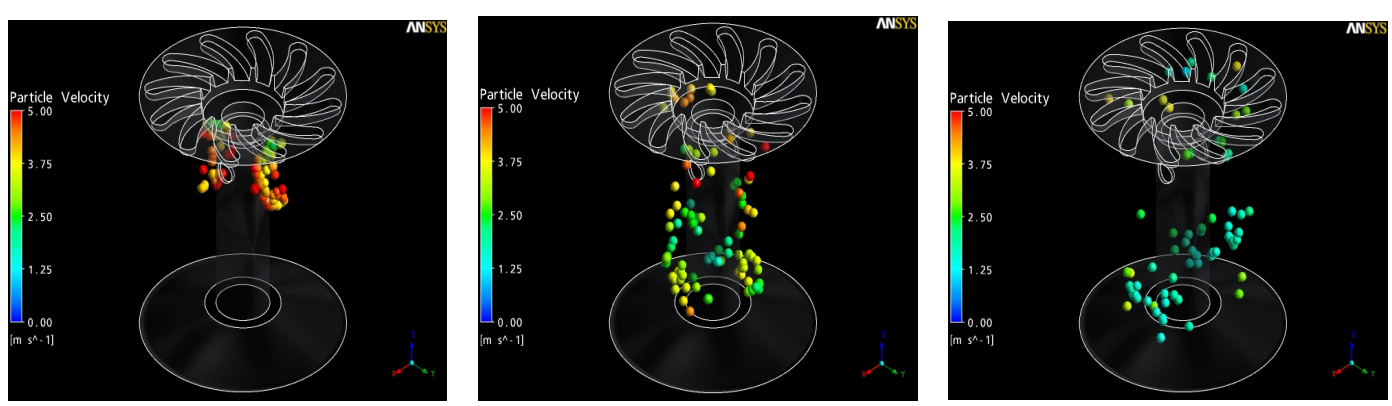

(c) 12 blade
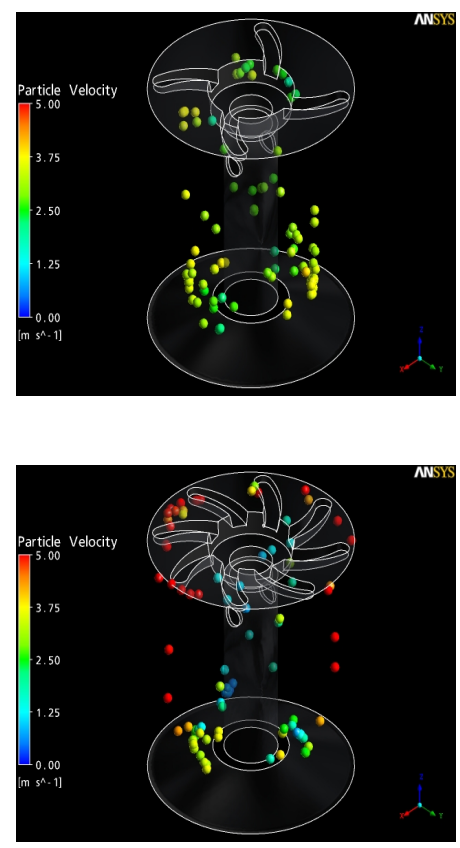

Figure 9: Physical behaviour of particles for the various models ( $\mathrm{N}: 3500 \mathrm{rpm}$, particle density: $6000 \mathrm{~kg} / \mathrm{m} 3$, particle size:30um) 
centrifugal pumps.

Figure 9 shows the particles' (impurity matters) movements for the three cases of blades. It can be seen from the figures that the particles are moving nearest the outer wall of the cylindrical chamber in case of 8-blade, which implies the highest filtration efficiency.

\section{Conclusion}

The characteristics of fluid flow and the physical behavior of the dust particles in a centrifugal oil purifier have been numerically investigated by using CFD simulations to estimate the filtration efficiencies.

The effects of particle size, particle density, and rotating speed of centrifugal filter have been examined. It have been found that the filtration efficiency increases as the particle size, particle density, and rotating speed of fluid increase. And the 8-blade case showed the highest efficiency among three cases along all particle sizes, although the effect reduces over the particle size 50um. For increasing filtration efficiency at the size smaller than $30 \mu \mathrm{m}$, additional studies and investigations are needed.

\section{Acknowledgement}

This research was supported by the second stage of BK21(Brain Korea21) of the Ministry of Education, Science and Technology, and partly by the National Research Lab. of Korea Science and Engineering Foundation(R0A-2008-000-20069-0).

\section{References}

[1] MANN+HUMMEL GMBH, Business
Unit Industrial Filters

[2] Sang-Hu, Jeong, “A study on the standard criteria of solid particle separation test for marine centrifugal purifier", Journal of the Korean Society of Marine Engineering, vol. 31, no. 8, pp. 1028-1034, 2007.

[3] I. Y. Lee and J. W. Kim, "A basic study on the integrated lubrication system for large scale marine diesel engines", Spring Conference of the Korean Society for Power System Engineering, pp. 262-266, 2003.

[4] Jin-Man Kim, Jun-Hee Lee, Yong-Kwan Yoon and Heuy-Dong Kim, "A study of the performance improvement of a centrifugal separator for gas-liquid two-phase flow", Proceedings of the KSME 2007 Spring Annual Meeting, pp. 3352-3357, 2007.

[5] K. H. Bang, K. K. Kim, Y. A. Song and P. S. Kim, "Numerical analysis of fluid flow and filtering efficiency in centrifugal oil filter", Journal of the Korean Society of Marine Engineering, vol. 33, no. 6, pp. 867-872, 2009.

[6] D. E. Smiles, "Centrifugal filtration of particulate systems", Chemical Engineering Science 54, pp. 215-224, 1999.

[7] Ansys CFX Release 11.0 manual, ANSYS Inc., 2006

[8] A. Oztekin, B. R. Seymour, E. Varley, "Pump flow solution of the navierStokes equations", Studies in Applied Math, 107, pp. 1-41, 2001

[9] A. J. Stepanoff, Centrifugal and Axial Flow Pumps(Theory, Design and Application), Krieger Pub Co., 2007. 


\section{Author Profile}

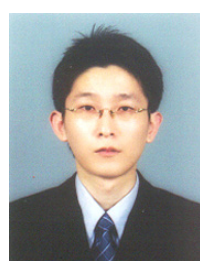

\section{Ho-Yun Jung}

He graduated from Pukyong National University (B.E. 2004, M.Sc. 2007) in Korea. At present, he has been studing as a Ph.D. student. School of Mechnical Engineering (CFD \& Energy Laboratory) in Pukyong National University, Busan, Korea.

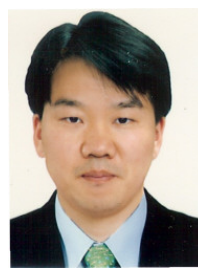

\section{Yoon-Hwan Choi}

He graduated from department of mechanical engineering of Dong-A university in Busan Korea(B. E. 1996, M. Sc. 1998, Ph.D. 2001). At present, he has been researching as a post Doc. of BK21 in Pukyong national university, Busan, Korea.

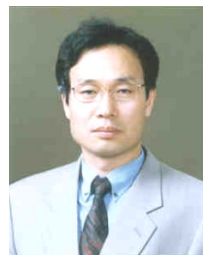

\section{Yeon-Won Lee}

He graduated from Kyungpook National University (B.E. 1981, M.S. 1983). He graduated from the Department of Mechanical Engineering of the University of Tokyo, Japan(PhD. 1993). He is a vice president of KOSME. He is one of the managing editors of JOV(Journal of Visualization). He is one of the editors of KSV(Korean Society of Visualization) journal.

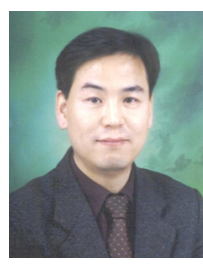

\section{Deog Hee Doh}

He graduated from Korea Maritime University((B.E., 1985, M.S. 1988). He graduated from the Department of Mechanical Engineering of the University of Tokyo, Japan(PhD. 1995). He is the vice editor of KSME journal(Fluid Engineering Department). He is one of the editors of the journal(Korean Fluid Machinery Association). His major research interests is to develop measurement techniques such as 4D-PIVs, 4D-PTVs for the analysis of turbulent flows. 